\title{
Design and Fabrication of sub-THz Steerable Photonic Transmitter $1 \times 4$ Array for Short-Distance Wireless Links
}

\author{
Luis Gonzalez Guerrero, Chris Graham, James \\ Seddon, Cyril Renaud, \\ Dept of Electronic \& Electrical Eng, UCL. \\ United Kingdom \\ luis.guerrero.14@ucl.ac.uk, c.renaud@ucl.ac.uk \\ Bilal Hussain, H. M. Salgado, L. M. Pessoa, \\ INESCTEC \\ Portugal \\ luis.m.pessoa@inesctec.pt
}

\author{
Glenn George, \\ Bay Photonics Ltd. \\ United Kingdom \\ glenn.george@bayphotonics.com
}

Alberto Hinojosa, Juan Fernandez, Marco A. G. Porcel VLC Photonics S.L.

Spain

marco.garcia@vlcphotonics.com
Abstract-Here we present the latest results on the design, fabrication and test of stand-alone photonic devices devoted to ultra-high bandwidth wireless access networks operating near the Terahertz (THz) band, combined with the demonstration of a hybrid photonic packaging of a $1 \times 4$ antenna array matching the $1 \times 4$ InP UTC-PDs array butt-coupled to a SiN PIC phase control array.

Keywords-Terahertz; Wireless communications; UTC-PDs; Beam steering; microwave photonic antenna; Photonic packaging.

\section{INTRODUCTION}

The saturation of wireless spectrum access is leading to technology innovations in areas such as spectrum resource usage. The millimetric frequency bands currently in use (below $100 \mathrm{GHz}$ ) do not seem sufficient to accommodate the predicted future data-rate requirements [1]. On the horizon beyond 4G and $5 \mathrm{G}$ networks, Terahertz wireless communications transceiver components will play a crucial role in ultra-fast data capacity wireless link oriented to data centre networks. Also behaving in real world short-range scenarios as offices or universities areas. This entails to satisfy extremely highly controlled environmental conditions, and significantly alleviating the workloads interconnections in this area.

Achieving the Tbit/s wireless connectivity paradigm requires the employment of very high frequency bands above $0.1 \mathrm{THz}$ and up to $10 \mathrm{THz}$. Submillimetre-wave radiation implies several challenges in wireless communications including harsher propagation conditions, channel suffering important absorption and multipath scattering and hardware limitations in terms of gain and spectral efficiency [2-3]. Photonic integration platforms such as Silicon Photonics, Silicon Nitride (SiN) and Indium Phosphide (InP) [4-5] are emerging as potential key technology while also adding the known benefit of miniaturization and cost reduction. Photonic applications are devoted not only for the radio-over-fibre growth but to enrich new industrial niches such as in $\mathrm{THz}$ applications [6-8], by taking advantage of its ultra-wide band frequency tuneability and natural convergence with optical networks.

This paper reviews the sub-THz photonic-based technology devices developed as part of TERAPOD project [9], comprising the radiative design of the high-frequency four element linear antenna array, the $1 \times 4$ integrated Uni-Travelling Carrier PhotoDiodes (UTC-PDs) array and the monolithically integrated photonic phase distribution system. We also report the suitability to assemble all those components in a robust smallform factor package.

\section{BEAM FORMING CONCEPT}

The TERAPOD project aims to provide the technology for future data centre ultra-fast wireless links. A crucial part of this approach is the capacity of such systems to find and establish radio links between devices.

Sub-Terahertz wireless communications require power sources and antennas properly designed to meet special requirements, such as bandwidth, efficiency, directivity and output power.

Beam forming systems bring a competitive advantage in $\mathrm{THz}$ wireless communications through spatial diversity by employing arrays of antenna elements combined with signal processing components. In addition to these, good power coupling efficiency between sources and antennas is essential to maximize the radiated low power levels achieved by the sources, and consequently increase the efficiency of the overall system. So far, the achieved output power is in the order of $1 \mathrm{~mW}$ at 300 GHz for UTC-PDs. Therefore, THz UTC-PD sources, integrated with antennas, are still at a research level, although some prototypes have been successfully demonstrated [10-13]

In order to optimize the performance and reduce the power consumption, TERAPOD is developing a beam forming system which allows efficient and low-power multi-device links by optically controlling the directionality of the antenna. Such

Deleted: for
Deleted: narrow beamwidths and
Commented [AHS1]: Re-phrase, please.
Deleted: together
Commented [AHS2]: Reformulation, please.
No radio broadcasting (like FM or AM)
Deleted: radio
Deleted: broadcasting
Deleted: modelling


optical control is based on photonic integrated circuits (PICs), which enables to coherently add the radiated $\mathrm{THz}$ signal in a specific direction while suppressing undesired directions.

The target radiated carrier frequency of the transmitter is above $100 \mathrm{GHz}$. The pitch between the optical inputs of each antenna element is $520 \mu \mathrm{m}$. With the presented configuration, it is possible to guide coherent radiation generated by two different lasers with a radiation frequency difference between 100 and $300 \mathrm{GHz}$. The guided radiation will be delayed and coupled to an array of InP antennas capable of radiating at frequencies around $300 \mathrm{GHz}$. The transmitter chip design for integration with the phase distribution PIC and substrate integrated antenna is detailed in the following section.

\section{DEVICES DESCRIPTION}

A. Substrate integrated sub-THz Antenna Array

In the sub-THz domain, the fabrication challenges of InP, namely the isotropic etching profile [14-16] and extreme thinning of substrate hinders the design of On-chip antennas. On the other hand, lossy transitions (due to dielectric and radiation losses) implemented in InP [17], further decreases the power efficiency of the sub-THz system. In order to overcome performance limitation due to fabrication challenges and improve power efficiency, an antenna array is designed to harness the power from multiple photodiodes (up to $1 \mathrm{~mW}$ at $300 \mathrm{GHz}$ [18]) along with the possibility of beam steering in space.

As mentioned earlier, InP conventionally requires additional fabrication steps such as microwave etching or substrate thinning for the realization of wave-guiding structures such as microstrip and grounded co-planar waveguide (GCPW). As the wavelength inside the InP substrate at the sub-THz region is in the order of $283 \mu \mathrm{m} @ 300 \mathrm{GHz}$, the needed substrate thickness is less than $50 \mu \mathrm{m}$ for the realization of a microstrip structure. Similar limitations exist for the physical dimensions of GCPW

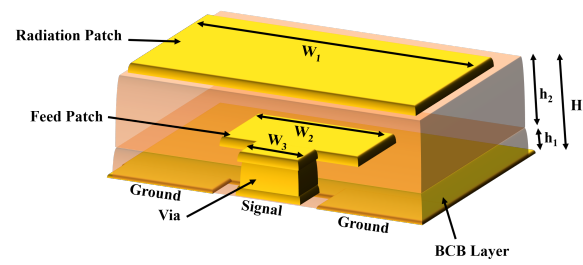

(a)

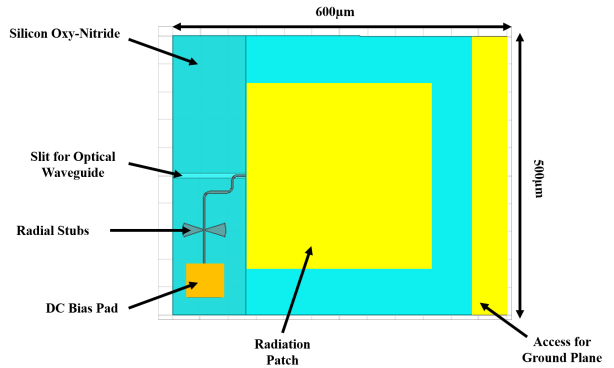

(b)

Fig. 1: (a) 3D view of single antenna element configuration and stack-up, (b) top view of finalized single antenna element.

structures. Instead of using $\mathrm{InP}$ as substrate, an additional organic dielectric layer of Benzcyclobutene (BCB) is deposited over the InP substrate.

Table 1: Dimension of Unit-cell shown in Fig. 1 (a), all dimensions in $\mu \mathrm{m}$
\begin{tabular}{|c|c|c|c|c|c|}
\hline $\mathbf{W}_{\mathbf{1}}$ & $\mathbf{W}_{\mathbf{2}}$ & $\mathbf{W}_{\mathbf{3}}$ & $\mathbf{h}_{\mathbf{1}}$ & $\mathbf{h}_{\mathbf{2}}$ & $\mathbf{H}$ \\
\hline 306 & 57.5 & 3 & 4 & 10 & 14 \\
\hline
\end{tabular}

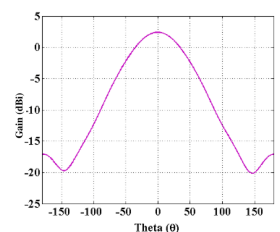

(a)

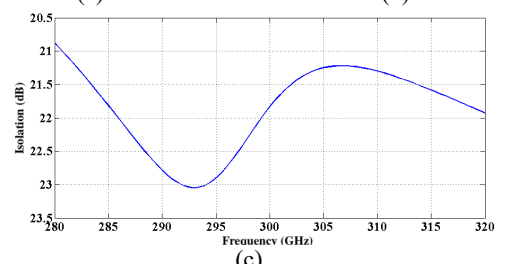

Fig. 2: (a) Simulated gain of Unitcell, (b) Return loss of Unit-cell, (c) isolation between DC-bias port and RF port.

The ground-signal-ground (GSG) output pads of the UTC-PDs are connected to a capacitively coupled antenna structure thereby eliminating the need of substrate thinning or plasma etching for the realization of via-holes. The proposed antenn structure is shown in Fig. 1 (a). The BCB layer is deposited over a metallic ground plane. The signal pad is connected to the capacitive feed using a via through the BCB layer with a heigh (h) of $4 \mu \mathrm{m}$. The choice of height is dictated by the cavity etching requirements of the $\mathrm{BCB}$ material. As the ground planes are not transported to the BCB layers, only a single via-hole is needed. A square patch is used as an antenna feed. The dimensions of the antenna feed are given in Table 1. As shown in Fig. 1 (a), a square shaped radiation patch is deposited ove the top of the BCB layer. It was demonstrated in [19] that the antenna efficiency is a function of the vertical distance between the radiation patch and feed patch. The higher the achieved isolation between radiation and feed patch, the higher the radiation efficiency becomes. On the other hand, the $\mathrm{BCB}$ layers tend to form cracks for thicknesses higher than $15 \mu \mathrm{m}$. Therefore, the distance between feed and radiation patches was kept at $10 \mu \mathrm{m}$. The total thickness $\left(\mathrm{H}=\mathrm{h}_{1}+\mathrm{h}_{2}\right)$ of the BCB layer is $14 \mu \mathrm{m}$. In order to provide a DC-bias to the photodiode, microstrip based bias line is implemented as shown in Fig. 1 (b).

The DC-bias line blocks the sub-THz signal at $300 \mathrm{GHz}$ by providing an isolation better than $20 \mathrm{~dB}$. The microstrip structure is implemented by depositing a Silicon-Oxynitride $\left(\mathrm{SiO}_{\mathrm{x}} \mathrm{N}_{\mathrm{y}}\right)$ layer $\left(500 \mathrm{~nm}\right.$ thickness). The added $\mathrm{SiO}_{\mathrm{x}} \mathrm{N}_{\mathrm{y}}$ layer provides strong adhesion between the BCB layer and metallic (gold) ground plane. A similar adhesion layer is deposited

\section{Commented [AHS4]: Reviewer comments:}

Fig. 2 should be on the same page where it is first references (page 3)

In addition, it would be good if vector graphics would be use for the curves.

It is also unclear how theta is defined in this case.

I assumed it is the same Theta as in Fig. 3. 
between the top BCB layer and the radiation patch. The biasing structure includes two radial stubs such that the impedance of the antenna element becomes the complex conjugate of the photodiode's output impedance. The simulated results of the unit-cell are presented in Fig. 2. The designed structure can provide a gain of $2.4 \mathrm{dBi}$ with a radiation efficiency of $46.7 \%$.

After finalizing the unit-cell, a $1 \times 4$ planar antenna array was created as shown in Fig. 3 (a). The planar one-dimensional design provides design flexibility for the optical routing. In the case of a $2 \mathrm{D}$ antenna array, the length of the optical waveguides cannot be uniform and thus will have varying optical path lengths. The array structure was simulated, and the results are presented in Fig. 3 (b). It can be observed that the array has the capability of beam steering over an angular range of $60^{\circ}$ in the broadside direction. The half power beam width is approximately $26^{\circ}$ and the side lobe levels (SLL) are better than $10 \mathrm{~dB}$ for the specified steering angles. Note that the limits of beam steering can be increased to $+/-90^{\circ}$ by compromising the SLL performance.

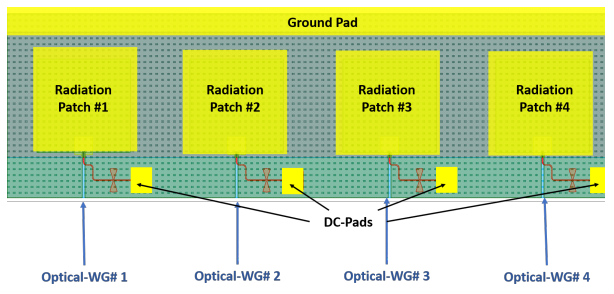

(a)

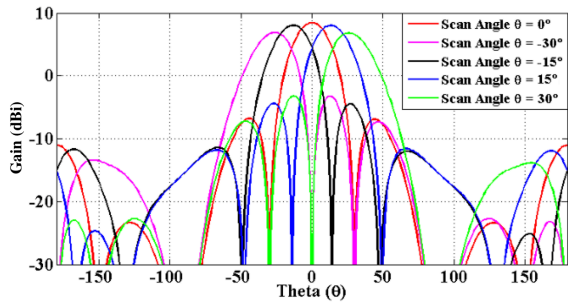

(b)

Fig. 3: (a) Finalized mask layout of $1 \times 4$ array, (b) Simulated gain of antenna array for different scan angles.

\section{B. UTC-PDs}

The antenna-integrated uni-travelling carrier (UTC) photodiode (PD) array is currently under fabrication at University College London. The UTCs being fabricated for the array module are waveguide integrated. Compared to vertically illuminated UTCs, waveguide integrated PDs have shown to ease the strong responsivity-bandwidth trade-off found in the former and have produced record output powers [20]. The UTC epitaxial structure - detailed in [21] - contains a $300 \mathrm{~nm}$ n-InP collection layer and a $300 \mathrm{~nm} \mathrm{n++-InGaAsP}$ waveguide layer.
A first round of UTCs with the same epitaxial structure and a bow tie antenna were fabricated in UCL for use as single emitters (i.e., not in an array). A picture of such UTCs is shown in Fig. 4. Micrograph of antenna coupled single emitter UTC. The measured leakage current was below $100 \mathrm{nA}$ at $1 \mathrm{~V}$ of negative bias and the responsivity was $0.16 \mathrm{~A} / \mathrm{W}$. Powers of 32 $\mu \mathrm{W}$ and $60 \mu \mathrm{W}$ (with respective photocurrents of $10 \mathrm{~mA}$ and $13.5 \mathrm{~mA}$ ) were measured at $250 \mathrm{GHz}$ from one of these UTC mounted in a $6 \mathrm{~mm}$ diameter Si lens [21]. The lower-thanexpected output powers have been attributed to inaccurate modelling parameters [22] which have now been refined by experimental measurement for this newer design.

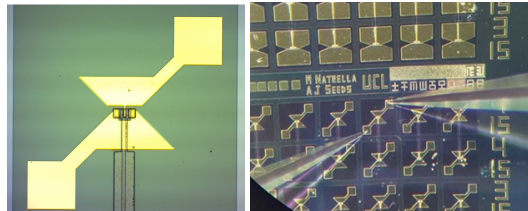

Fig. 4. Micrograph of antenna coupled single emitter UTC

C. Optical Phase Array Beam Steering

Exploiting the photonic integration technology for terahertz systems, VLC Photonics has developed a phase distribution photonic integrated circuit to control the input laser light to four different outputs, which will be coupled into an array of fou antenna elements developed by UCL and INESC TEC. The design of a phase distribution PIC for $100-300 \mathrm{GHz}$ signals will provide beam steering capabilities.

The phase distributed array consists of a fully integrated 2-stage power splitter with phase control. Firstly, the power is divided into four outputs by means of a 2-stage Mach-Zehnder interferometer (MZI)-based power splitter. The MZIs are thermally controlled, which allows the antenna array radiation pattern to be modified, with $\mathrm{kHz}$ switching speeds. Finally, the pointing direction of the radiation pattern is controlled by the delay of the signal towards each antenna element by means of series of micro-ring resonators [23], providing together a flat response in the delay along a given spectral bandwidth for truetime delay (TTD) operation.

The phase distribution system based on ring resonators is depicted in a GDS representation of the fabricated PIC system design presented in Fig. 5. Layout illustration of the TERAPOD phase distribution PIC. Die area $5 \times 10 \mathrm{~mm}^{2}$. The central area encompasses the phase-distribution system plus the alignment waveguides. The latest design included some test structures at north (top) and south (bottom) areas of the PIC. These structures are used within the full system and by including them independently in the die, we can characterize their overall performance directly.

The phase distribution PIC was fabricated on the low-loss SiN industrial photonic Damascene process AN800 at Ligentec. The layout contains an Aluminium standard metallization laye compatible with CMOS manufacturing. The metal layer is required for the control of the beam steering by means of micro- 
heating elements. To ease the packaging, each element is wired towards the north or southedge of the layout and ended in a 100 $\mu \mathrm{m}^{2}$ pads and spaced centre to centre with a pitch of $250 \mu \mathrm{m}$

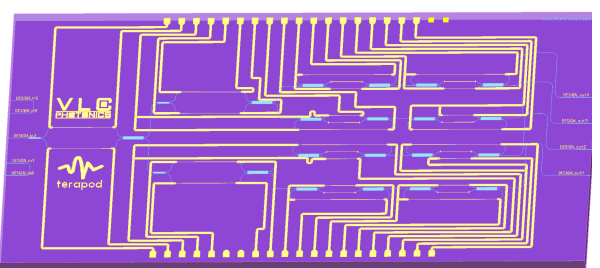

Fig. 5. Layout illustration of the TERAPOD phase distribution PIC. Die are $5 \times 10 \mathrm{~mm}^{2}$

With the aim to provide a true-time delay over the broaden spectra of modulated signals, the phase tuneable stage consists of two cascaded ring resonators. Looking to improve the accuracy of the tuneable delay, preserving a safe and functional accuracy of the tuneable delay, preserving a safe and functional ring resonators, the widths of the heaters were doubled from $1 \mu \mathrm{m}$ to $2 \mu \mathrm{m}[24]$.

In order to provide efficient coupling of light from the PIC to the waveguides on the UTC-PDs, the optical mode overlap was maximized by enlarging the width of the $\mathrm{SiN}$ waveguides at the output optical ports matching the optical mode at the UTC-PD waveguide. Fig. 6 shows the output waveguides width that was enlarged to $4.225 \mu \mathrm{m}$, leading a mode field diameter (MFD) of $3.4 \mu \mathrm{m}$ in the $\mathrm{X}$-direction and $0.91 \mu \mathrm{m}$ in the Y-direction for fundamental quasi-TE mode.

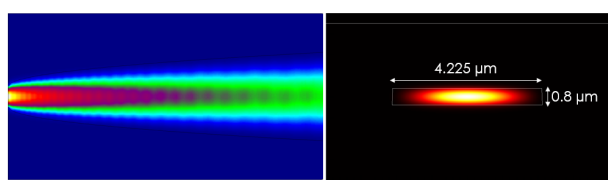

Fig. 6. Propagation simulation and single-mode beam of the output waveguide tapering.

Characterization of building blocks show the expected performance. The 50:50 multimode interference (MMI) splitters exhibit an imbalance below $2 \%$ along a bandwidth of $40 \mathrm{~nm}$, presenting excess losses up to $0.3 \mathrm{~dB}$ for fundamental quasi-TE mode. The MZIs for power switching operate in a current-bias range of $35 \mathrm{~mA}$ (between 30 to $75 \mathrm{~mA}$ ) to tune the splitting ratio at the outputs. The MZI-based ring resonators have heating elements to control the coupling. The resonance can be varied from over-coupled to critically coupled, achieving a maximum extinction ratio of above $5 \mathrm{~dB}$ in critical coupling (at $65 \mathrm{~mA}$ ) on each ring independently. This corresponds to a phase delay of the sub-THz signal from 0 to $12 \pi$ rad. The optical spectral response is depicted in Fig. 7
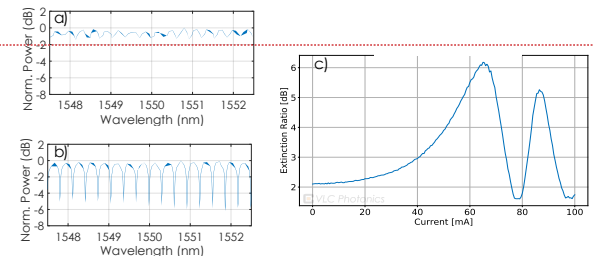

Fig. 7: Spectral response coupling operation, respectively. (c) Extinction ratio by current steps of $1 \mathrm{~mA}$ at $1550 \mathrm{~nm}$.

In summary, the phase control and delay lines are constructed with cascaded all-pass filters based on ring resonator cavities. The delay introduced by the micro-ring in critical coupling has been shown to be sufficient for a 100 to $300 \mathrm{GHz}$ signal to accomplish $\pm \pi / 4 \mathrm{rad}$ beam steering. Besides, the True-TimeDelay provided by the double ring structure can improve the bandwidth of a 100 to $300 \mathrm{GHz}$ signal and to accomplish signal radiation beam steering.

\section{HYBRID PACKAGING}

The final packaged devices with integrated multiple modules and components for the show-case demonstration have been constructed at Bay Photonics Ltd in UK.

The main difficulty in achieving integration of the component was the mechanical adjustment of the 1-in and 4-output channels of the waveguide PIC with the lens mounted together with the 4-chip UTC bar while also the mutual aligning dependence between the single input PIC to the $9 \mu \mathrm{m}$ core single mode fibre.

This has been achieved by constructing 2 sub-assemblies and combining them in the package assembly. First the 4-chip UTC bar is mounted centrally on the Hyper-spherical millimetrewave lens along with a small tracking sub-mount to allow wirebond connection to the UTCs as shown in Fig. 8.

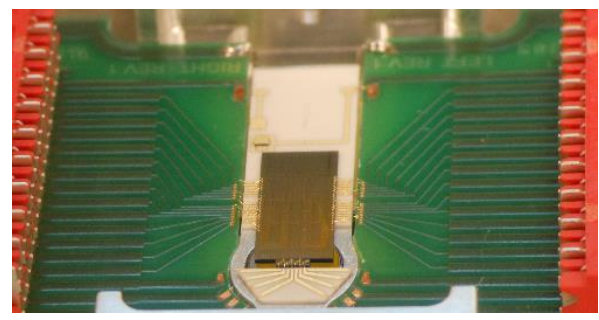

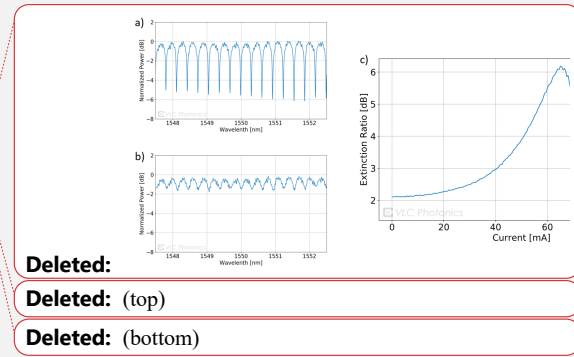

Deleted: (bottom) 


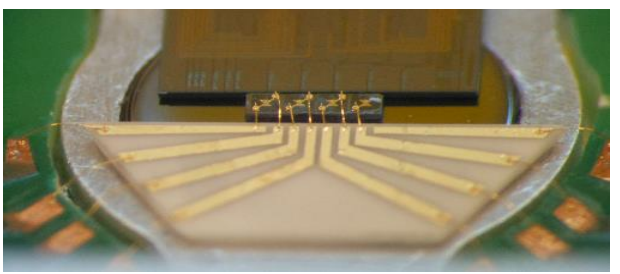

(b)

Fig. 8: A picture of the (a) opto-electrical photonic assembly. (b) Inset of the 4-chip UTC bar aligned in front of the 4-output waveguide PIC's channels. The whole set is placed on the millimetre-wave lens.

Separately the PIC array is mounted on a carrier with space to allow the optical fibre to be attached and actively aligned after the 2 sub-assemblies have been aligned in the package. Fig. 9 emphasises the protrusion of the PIC attached in the ceramic mount. A further complication is the height difference between the waveguide output of the $1 \times 4$ PIC and the active region on the UTCs. This was reconciled by selective back grinding of the PIC to provide a step which fits over the lens and delivers an optimal optical alignment.

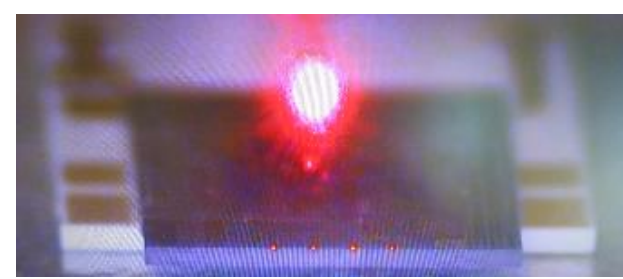

Fig. 9: $1 \times 4$ PIC on the ceramic mount. Visual inspection during single-mode fibre optical alignment by injecting red-light to identify the output channels.

\section{CONCLUSION}

In this article we presented a Terahertz wireless communication packaged device developed in the TERAPOD project. We detailed the design, fabrication, and characterization of the photonic and sub-THz band discrete components. A photonic phased-array distribution network is proposed for beamsteering, UTC-PDs for optical-to-THz signal conversion and a $1 \times 4$ planar antenna array for efficient radiation. The performance of the components is promising to operate at around 100 to $300 \mathrm{GHz}$. Finally, we report the hybrid packaging, assembling the components together with a millimetre-wave lens in a form-factor housing. The assembled parts are to be tested for performance at UCL and National Physical Laboratory (NPL) facilities in London, UK.

\section{ACKNOWLEDGMENT}

This project has received funding from the European Union's Horizon 2020 research and innovation programme under grant agreement 761579 (TERAPOD).

\section{REFERENCES}

[1] Kürner, T., Priebe, S. Towards THz Communications - Status in Research, Standardization and Regulation. J Infrared Milli Teraher

[2] Zhao, J., Li, C., Li, X. et al. Technical challenges for high-frequency wireless communication. J. Comm
https://doi.org/10.1007/BF03391554

[3] Yuan, Y., Zhao, Y., Zong, B. et al. Potential key technologies for $6 \mathrm{G}$ f. Sci. 63, $183301 \quad(2020)$ https://doi.org/10.1007/s11432-019-2789-y

[4] C. Zhu, L. Lu, W. Shan, W. Xu, G. Zhou, L. Zhou, and J. Chen, "Silicon integrated microwave photonic beamformer," Optica 7, 1162-11 (2020). https://doi.org/10.1364/OPTICA.391521

[5] Carpintero, G., Hisatake, S., de Felipe, D. et al. Wireless Data Transmission at Terahertz Carrier Waves Generated from a Hybrid InPPolymer Dual Tunable DBR Laser Photonic Integrated Circuit. Sci Rep 8 , 3018 (2018). https://doi.org/10.1038/s41598-018-21391-0

[6] Sengupta, K., Nagatsuma, T. \& Mittleman, D.M. Terahertz integrated electronic and hybrid electronic-photonic systems. Nat Electron 1, 622 635 (2018). https://doi.org/10.1038/s41928-018-0173-2

[7] Maurizio Burla, David Marpaung, Leimeng Zhuang, Chris Roeloffzen, Muhammad Rezaul Khan, Arne Leinse, Marcel Hoekman, and Ren Heideman, "On-chip CMOS compatible reconfigurable optical delay line with separate carrier tuning for microwave photonic signal processing," Opt. Express 19, 21475-21484 (2011)

[8] M. Burla et al., "Integrated Photonic-Band Beamformer Chip With Technology Letters, vol. 25, no. 12, pp. 1145-1148, June15, 2013.

[9] Horizon 2020 Terahertz Based Ultra High Bandwidth Wireless Access Networks (TERAPOD) https://terapod-project.eu/

[10] K. Okada, K. Kasagi, N. Oshima, S. Suzuki, and M. Asada, "Resonant-Tunneling-Diode Terahertz Oscillator Using Patch Antenna "Resonant-Tunneling-Diode Terahertz Oscillator Using Patch Antenna
Integrated on Slot Resonator for Power Radiation," IEEE Trans. Terahertz Sci. Technol., vol. 5, no. 4, pp. 613-618, 2015.

[11] K. Okada, S. Suzuki, and M. Asada, "Resonant-tunneling-diodeterahertz oscillator integrated with slot-coupled patch antenna," Conf. Proc. -Int. Conf. Indium Phosphide Relat. Mater., pp. 14-15, 2014.

[12] Y. Koyama, R. Sekiguchi, and T. Ouchi, "Oscillations up to1.40 THz from resonant-tunneling-diode-based oscillators with integrated patc

[13] E. Rouvalis, C. C. Renaud, D. G. Moodie, M. J. Robertson, and A. J. Seeds, "Traveling-wave Uni-Traveling Carrier Photodiodes for continuous wave THz generation," Opt. Express, vol. 18, no. 11, p. 11105 , 2010 [14] C. Constantine et al., "Dry etching of via connections for InP power
devices," Electron. Lett., vol. 29, no. 11, Art. no. 11, May 1993, doi: 10.1049/el: 19930655 .

[15] R. Khare, " $\mathrm{CH} 4 / \mathrm{H} 2 / \mathrm{Ar} / \mathrm{Cl} 2$ electron cyclotron resonance plasma etching of via holes for InP-based microwave devices,” J. Vac. Sci. Technol. B Microelectron. Nanometer Struct., vol. 12, no. 5, Art. no. 5, Sep. 1994, doi: $10.1116 / 1.587541$

[16] S. Trassaert, "Bromine/methanol wet chemical etching of via holes for InP microwave devices," J. Vac. Sci. Technol. B Microelectron. Nanometer Struct., vol. 16, no. 2, Art. no. 2, Mar. 1998, doi: $10.1116 / 1.589863$

[17] B. Hussain, G. Serafino, P. Ghelfi, A. Bogoni, and A. Stohr, "Via-Less Microstrip to Rectangular Waveguide Transition on InP," in 2019 44th International Conference on Infrared, Millimeter, and Terahertz Waves (IRMMW-THz), Paris, France, Sep. 2019, pp. 1-2, doi: 10.1109/IRMMW-THz.2019.8874316.

[18] C. C. Renaud, M. J. Fice, L. Ponnampalam, M. Natrella, C. Graham, and A. J. Seeds, "Uni-travelling carrier photodetectors as THz detectors and emitters," San Francisco, C

[19] E. Rouvalis, M. J. Robertson, and A. J. Seeds, "Continuous Wave E. Rouvalis, M. J. Robertson, and A. J. Seeds, "Continuous Wave
Terahertz Generation From Ultra-Fast InP-Based Photodiodes," IEEE Trans. Microw. THEORY Tech., vol. 60, no. 3, p. 9, Phot 
[20] A. J. Seeds, H. Shams, M. J. Fice and C. C. Renaud, "TeraHertz Photonics for Wireless Communications," in Journal of Lightwave Technology, vol. 21] X. Lin, M. Natrella, J. Seddon, C. Graham, C. C. Renaud, M. Tang, J. Wu, H. Liu, and A. J. Seeds, "High performance waveguide uni-travelling carrier photodiode grown by solid source molecular beam epitaxy," Opt. Express, vol.

[22] M. Natrella, C.-P. Liu, C. Graham, F. van Dijk, H. Liu, C. C. Renaud, and A. J. Seeds, "Accurate equivalent circuit model for millimetre-wave UTC photodiodes," Opt. Express 24(5), 4698-4713 (2016).
[23] Liu X, Kolpatzeck K, Häring L, Balzer JC, Czylwik A. Wideband Beam Steering Concept for Terahertz Time-Domain Spectroscopy: Theoretica Considerations. Sensors (Basel). 2020 Sep 28,20(19).5568. doi:

24] D. Pérez, J. Fernández, R. Baños, J.D. Doménech, A.M. Sánchez, J.M. Cirera, R. Mas, J. Sánchez, S. Durán, E. Pardo, C. Domínguez, D. Pastor $J$. Capmany and P. Munoz, Thermal tuners on a Silicon Nitride platform," 2016 arXiv:1604.02958 [physics.optics] 